\title{
Avaliação da perda de inserção dentária em pacientes com doença renal crônica em hemodiálise
}

\author{
Evaluation of dental insertion loss in patients with chronic kidney \\ disease
}

\begin{abstract}
Autores
Érica Melo Gonçalves ${ }^{1}$

Danilo Lopes Ferreira Lima $^{1,2}$

Sandra Helena C. de Albuquerque ${ }^{1}$

José Arthur de

Carvalho ${ }^{1}$

Tiago França Araripe

Cariri ${ }^{1}$

Cláudia Maria Costa

de Oliveira ${ }^{3}$

${ }^{1}$ Universidade de

Fortaleza - UNIFOR.

${ }^{2}$ Universidade Federal do

Rio Grande do Norte -

UFRN.

${ }^{3}$ Faculdade de Medicina

Christus.
\end{abstract}

Data de submissão: 25/04/2010

Data de aprovação: 03/03/2011

\section{Correspondência para: \\ Érica Melo Gonçalves Universidade de Fortaleza \\ Rua Carolina Sucupira, \\ 444 - Aldeota \\ Fortaleza (CE) - Brasil \\ CEP: $60140-120$ \\ E-mail: \\ ericamelogoncalves@ \\ yahoo.com.br}

$O$ referido estudo foi realizado na Clínica de Hemodiálise Prontorim, Fortaleza (CE) - Brasil.

Os autores declaram a inexistência de conflitos de interesse.

\section{Resumo}

A doença renal crônica (DRC) é uma síndrome clínica decorrente da perda lenta, progressiva e irreversível da taxa de filtração glomerular. A DRC pode apresentar várias manifestações bucais, como xerostomia, estomatite urêmica e periodontite, diagnosticada por meio da perda de inserção clínica (PIC). Foram avaliados 92 indivíduos em tratamento de hemodiálise na Clínica de Hemodiálise Prontorim, na cidade de Fortaleza, Ceará. O exame periodontal foi realizado por meio da mensuração da PIC e apenas 34 pacientes (37\%) estavam aptos a realizar exame periodontal. A perda de inserção por indivíduo registrada no grupo de dentados ficou entre 1,31 e 5,27 mm, com média de 2,30 $\pm 0,96 \mathrm{~mm}$. Dezoito pacientes $(52,9 \%)$ apresentaram PIC menor que $2 \mathrm{~mm}$, enquanto $16(47,1 \%)$ mostraram-se portadores de periodontite. A perda dentária e a presença de considerável perda de inserção foram observadas neste estudo. Contudo, fatores como status social devem ser considerados em futuras investigações. Palavras-chave: Periodontite. Diálise renal. Insuficiência renal.

\section{Abstract}

Chronic kidney failure (CKF) is a clinical syndrome due to the slow, progressive and irreversible loss of the glomerular filtration rate. CKF may be associated with several oral manifestations, such as xerostomia, uremic stomatitis and periodontitis, diagnosed as clinical attachment loss (CAL). 92 patients, from the Prontorim Hemodialysis Clinic of Fortaleza, Ceará, Brazil, were investigated. Only 34 patients $(37 \%)$ were eligible for periodontal investigation, which consisted of CAL measurement (values grater than $2 \mathrm{~mm}$ diagnosing periodontitis). CAL measurements ranged from 1.31 to $5.27 \mathrm{~mm}$ $(2.30 \pm 0.96 \mathrm{~mm}) .18(52.9 \%)$ patients had smaller than $2 \mathrm{~mm}$ CAL, while 16 $(47.1 \%)$ had periodontitis. Tooth loss and considerable CAL were observed in this study. Nevertheless, factors such as social status should be considered in further investigations.

Keywords: Periodontitis. Renal dialysis. Renal insufficiency.

\section{INTRODUÇÃO}

A doença renal crônica (DRC) é uma síndrome clínica decorrente da perda lenta, progressiva e irreversível da taxa de filtração glomerular. ${ }^{1}$ São várias as causas de DRC, estando hipertensão arterial, glomerulonefrites e diabetes mellitus como os principais fatores etiológicos. A história familiar de DRC é considerada um fator de risco elevado juntamente com doenças cardiovasculares. ${ }^{1,2}$

No Brasil, ainda não dispomos de dados confiáveis sobre a incidência e prevalência de DRC, uma vez que o diagnóstico precoce é difícil, as medidas preventivas são escassas e frequentemente a doença é diagnosticada em seu estágio terminal. Segundo dados do Censo da Sociedade Brasileira de Nefrologia $(\mathrm{SBN}),{ }^{3}$ das 626 unidades de diálise cadastradas na sociedade, $437(69,8 \%)$ responderam ao censo, sendo que quase metade destas estavam na região Sudeste $(49,2 \%)$. Em janeiro de 2009, o número estimado de pacientes em diálise foi de 77.589. As estimativas das taxas de prevalência e de incidência de insuficiência renal crônica em tratamento 
dialítico foram de 405 e 144 pacientes por milhão da população, respectivamente. O número estimado de pacientes que iniciaram tratamento em 2009 foi de 27.612. A taxa anual de mortalidade bruta foi de $17,1 \%$. Dos pacientes, $39,9 \%$ tinham idade maior ou igual a 60 anos, $89,6 \%$ estavam em hemodiálise e 10,4\% em diálise peritoneal; $30.419(39,2 \%)$ estavam em fila de espera para transplante e $27 \%$ eram diabéticos.

A DRC pode apresentar várias manifestações bucais como xerostomia, estomatite urêmica, alterações radiográficas dos ossos maxilares e mandibulares secundárias à perda de cálcio do tecido ósseo devido ao aumento do paratormônio (perda da lâmina dura e lesões radiolucentes), formação de cálculo dentário decorrente do aumento da concentração de cálcio e fósforo séricos, alta concentração de ureia na saliva, remodelamento ósseo anormal após extração, erupção dentária atrasada, baixa prevalência de cáries, mobilidade dentária, maloclusão, sensibilidade à percussão e mastigação, entre outras. ${ }^{4,5} \mathrm{~A}$ doença periodontal também se mostrou prevalente em portadores de $\mathrm{DRC}^{6} \mathrm{e}$ pode ser dividida em dois diferentes grupos: gengivites e periodontites. As gengivites caracterizam-se por eritema, edema e alargamento do tecido gengival, e são reversíveis com o tratamento. A periodontite caracterizase pela reabsorção do osso alveolar que dá suporte aos dentes. Os micro-organismos anaeróbicos gram-negativos são a principal causa da perda óssea. A presença da bolsa periodontal é o sinal chave para o diagnóstico da periodontite e, em muitos casos, ocasiona mobilidade dentária. ${ }^{7}$ A prevalência e a severidade da periodontite têm sido descritas como sendo mais prevalentes em pacientes renais crônicos do que na população em geral. $^{8-10}$ A periodontite tem sido associada a uma resposta inflamatória sistêmica aumentada na população geral, e a análise dos dados do National Health and Nutrition Examination Survey III (NHANES III) encontrou uma associação positiva entre níveis elevados de marcadores do estado inflamatório, como proteína C-reativa, e severidade da doença periodontal. ${ }^{11} \mathrm{O}$ presente estudo objetivou avaliar a condição periodontal em pacientes com DRC por meio da verificação da perda de inserção clínica.

\section{Materials e métodos}

Este estudo apresenta o desenho de um corte transversal. Foram avaliados 92 indivíduos em tratamento de hemodiálise na Clínica de Hemodiálise Prontorim, na cidade de Fortaleza, Ceará.

Em pacientes com menos de 10 unidades dentárias, não foi realizado o exame periodontal. Após o acesso ao banco de dados e prontuários dos pacientes, foram registrados os seguintes dados:

- características demográficas: idade e sexo;

- hábitos: tabagismo;

- co-morbidades: diabetes mellitus.

A avaliação dentária foi feita com a utilização dos seguintes instrumentos: sonda periodontal UNC-15 (Hartzell, CA, USA), pinça clínica e espelho bucal. O material foi esterilizado em autoclave, e o exame foi realizado de acordo com as normas prescritas pela Comissão de Biossegurança da Universidade de Fortaleza (UNIFOR).

O exame periodontal foi realizado por meio da mensuração da perda de inserção clínica (PIC) em 3 pontos: vestibular, mesiovestibular e distovestibular. A PIC para cada superfície foi obtida por meio da profundidade de sondagem (PS) menos a distância da junção cemento-esmalte (JCE) à margem gengival se a esta for coronária à JCE - ou PS mais a distância entre a JCE e a margem gengival se esta for apical à JCE. Para PIC a partir de uma média de $2 \mathrm{~mm}$, considerouse presença de periodontite. Os resultados foram expressos em média \pm desvio padrão.

Este projeto de pesquisa foi cadastrado no Sistema Nacional de Informação sobre Ética em Pesquisa (SISNEP) e submetido ao Comitê de Ética da UNIFOR (COÉTICA) para apreciação e aprovação, segundo a resolução 196/96 do Conselho Nacional de Saúde (CNS), que determina as diretrizes e normas regulamentadoras de pesquisas envolvendo seres humanos. A aprovação deu-se por meio do parecer $\mathrm{n}^{\circ}$ 050/2008.

\section{Resultados}

Dos 92 pacientes investigados, 20 (21,7\%) estavam impossibilitados de realizar exame periodontal ou se recusaram a participar do presente estudo, 15 (16,3\%) estavam fora do perfil por possuir menos de 10 unidades dentárias, $23(25 \%)$ eram desdentados totais e 34 (37\%) estavam aptos a realizar exame periodontal (Tabela).

\section{Tabela}

QUANDO GERAL DOS PACIENTES INVESTIGADOS

\begin{tabular}{lcc} 
Pacientes & Número & $\begin{array}{c}\text { Frequência } \\
(\%)\end{array}$ \\
\hline Pacientes dentro do perfil & 34 & 37,0 \\
Pacientes fora do perfil & 15 & 16,3 \\
Pacientes desdentados totais & 23 & 25,0 \\
Pacientes impossibilitados & 20 & 21,7 \\
Total & 92 & 100,0 \\
\hline
\end{tabular}


O grupo de pacientes desdentados e fora do perfil por possuírem menos de 10 unidades dentárias foi composto por 38 indivíduos, sendo $20(52,6 \%) \mathrm{mu}-$ lheres e $18(47,4 \%)$ homens. As idades variaram entre 35 e 96 anos, com média de 63,6 $\pm 16,5$ anos. O tempo de diálise verificado foi de 4,1 $\pm 3,1$ anos. Destes, $28(73,7 \%)$ não eram diabéticos e $10(26,3 \%)$ eram diabéticos. Os pacientes que são ou foram fumantes totalizaram $12(31,57 \%)$, enquanto $26(68,43 \%)$ não fumavam.

O grupo de pacientes dentados que estavam dentro do perfil e participaram da pesquisa foi composto por 34 indivíduos, dos quais $10(29,4 \%)$ eram mulheres e $24(70,6 \%)$ eram homens. O número de dentes presentes variou entre 12 e 28, com média de 21,18 $\pm 5,17$ dentes. Os pacientes tinham idades que variavam entre 23 e 68 anos, com média de 41,9 $\pm 12,5$ anos. A média de tempo de diálise verificada foi de 4,2 $\pm 3,9$ anos. Encontraram-se dentro do grupo um total de $3(8,8 \%)$ pacientes diabéticos e $31(91,2 \%)$ não diabéticos. Observaram-se no grupo 5 (14,7\%) fumantes e $29(85,3 \%)$ não fumantes.

A perda de inserção por indivíduo registrada no grupo de dentados ficou entre 1,31 e $5,27 \mathrm{~mm}$, com média de 2,30 $\pm 0,96 \mathrm{~mm}$. Um total de $18(52,9 \%)$ pacientes apresentaram perda de inserção clínica menor que $2 \mathrm{~mm}$, enquanto $16(47,1 \%)$ mostraram-se portadores de periodontite. Quando verificados todos os 688 sítios, observou-se que $338(49,12 \%)$ apresentaram perda de inserção $>2 \mathrm{~mm}, 196(28,48 \%)$ $>1 \mathrm{~mm}$ e somente 154 sítios $(22,4 \%)$ apresentaram perda de inserção $\leq 1 \mathrm{~mm}$.

\section{Dıscussão}

A perspectiva de que as periodontopatias podem ser fatores de risco para alterações sistêmicas fez com que extensivas pesquisas começassem a ser realizadas a partir do final dos anos 1980 . O trabalho realizado em $1989^{12}$ por pesquisadores finlandeses - relacionando as más condições da saúde dentária ao infarto agudo do miocárdio - foi o marco do retorno da teoria da infecção focal às pesquisas na Odontologia.

Seguindo esse raciocínio, pesquisadores realizaram estudos relacionando as doenças periodontais a várias alterações sistêmicas, como doenças cardiovasculares isquêmicas, ${ }^{13,14}$ doenças pulmonares, ${ }^{15,16}$ nascimento de bebês prematuros e com baixo peso, ${ }^{17,18}$ osteoporose ${ }^{19,20}$ e diabetes..$^{21,22}$ Assim, foi consolidada uma nova linha de pesquisa, fazendo com que - durante o Workshop Mundial de Periodontia realizado em 1996 - fosse introduzido o termo "Medicina periodontal" como uma área de estudo que tivesse como foco a plausibilidade biológica dessas relações.

Proteína C-reativa e imunoglobulinas, notadamente IgG, são mediadores inflamatórios produzidos durante as reações bacterianas no periodonto e que podem causar danos aos rins. Da mesma forma, as próprias bactérias catalogadas como periodontopatogênicas podem agir à distância e atingir órgãos vitais através da corrente sanguínea e, dentre estes, os rins podem estar incluídos. ${ }^{10}$

Estudos recentes têm demonstrado a associação entre as doenças renais crônicas e as doenças que acometem o periodonto. $\mathrm{O}$ acúmulo de cálculo em pacientes renais em hemodiálise ocorre em um nível acelerado, e acredita-se que esteja relacionado a um produto cálcio-fosfato sérico alterado. Gengivite também pode ser um achado comum em pacientes com DRC - a gengiva marginal é frequentemente inflamada com tendência à hemorragia e outras manifestações orais incluem a periodontite.

A grande presença de pacientes portadores de perda de inserção periodontal observada em nosso estudo vem demonstrar que, pelo menos, os hábitos de higiene oral daqueles com DRC submetidos à hemodiálise devem estar deficientes. Ficou evidenciada a falta de cuidado com a dentição nos resultados obtidos, visto que $41,30 \%$ dos avaliados eram desdentados totais ou possuíam menos de 10 unidades dentárias e a causa dessa perda dentária pode ter ocorrido por problemas periodontais.

Dois fatores de risco comuns às duas patologias foram observados: a idade avançada e o diabetes mellitus. O processo de envelhecimento não é responsável pelo aparecimento de doenças relacionadas à saúde bucal, mas estas estão geralmente presentes em indivíduos idosos. ${ }^{23} \mathrm{~A}$ doença do periodonto ocorre na infância, adolescência e juventude, mas a prevalência, a destruição tecidual e a perda dentária causadas pela doença periodontal aumentam com a idade. Muitas mudanças teciduais ocorrem com o envelhecimento, e algumas delas podem afetar as doenças periodontais. De fato, muitas vezes é difícil diferenciar entre o envelhecimento fisiológico e os efeitos acumulativos da doença. No presente estudo, verificou-se que o número de dentes perdidos, bem como o número de pacientes desdentados totais aumentou proporcionalmente com a idade. A perda de inserção também mostrou ser maior em pacientes mais velhos.

A literatura também relata que a situação dentária e periodontal piora com maior tempo de diálise; contudo, este estudo observou que tanto o grupo de 
pior situação dentária como o grupo dentado apresentaram médias de tempo de diálise semelhantes. ${ }^{24}$

O diabetes mellitus e a periodontite são patologias que estabelecem entre si influências mútuas, exigindo do profissional clínico um amplo conhecimento para conduzir a heterogeneidade dos diversos casos clínicos que se apresentam. Com a presença do diabetes, na dependência de tipo, tempo de instalação e eficiência do controle metabólico, uma série de manifestações bucais podem ocorrer, inclusive a periodontite, que estabelece uma inter-relação clara e estabelecida. ${ }^{25}$

O fato de a DRC ser causada tanto pela presença de diabetes mellitus como por processos infecciosos, através das glomerulonefrites, faz com que as duas patologias possam ter alguma ligação. Em recente estudo de correlação entre periodontite, nefropatia e diabetes tipo 2 , verificou-se que a periodontite prediz o desenvolvimento de doença renal em diabéticos tipo $2 .^{26}$

\section{Conclusão}

A perda dentária e a presença de considerável perda de inserção foram observadas neste estudo. Contudo, fatores como status social devem ser considerados em futuras investigações.

\section{RefERÊNCIAS}

1. Cuppari L. Insuficiência renal crônica. In: Cupari L. Guia de Nutrição: Nutrição clinica no adulto. Barueri: Manole; 2002. p. 167-92.

2. Ashwini R, Sehgal MD. Doença renal terminal: causas e conseqüências. Epidemiologia e resultados da doença renal terminal. In: Hricik DE, Sedor JR, Ganz MB. Segredos em Nefrologia. Porto Alegre: Artmed; 2002. p. 177-8.

3. Sesso RCC, Lopes AA, Thomé FS, Lungon JR, Burdmann EA. Censo Brasileiro de Diálise. J Bras Nefrol 2010;32:380-4.

4. De Rossi SS, Glick M. Dental considerations for the patient with renal disease receiving hemodialysis. J Am Dent Assoc 1996;127:211-9.

5. Proctor R, Kumar N, Stein A, Moles D, Porter S. Oral and dental aspects of chronic renal failure. J Dent Res 2005;84:199-208.

6. Borawski J, Wilczyńska-Borawska M, Stokowska W, Myśliwiec M. The periodontal status of pre-dialysis chronic kidney disease and maintenance dialysis patients. Nephrol Dial Transplant 2007;22:457-64.

7. Armitage GC. Development of a classification system for periodontal diseases and conditions. Ann Periodontol 1999;4:1-6.

8. Naugle K, Darby ML, Bauman DB, Lineberger LT, Powers R. The oral health status of individuals on renal dialysis. Ann Periodontol 1998;3:197-205.

9. Chen LP, Chiang CK, Chan CP, Hung KY, Huang CS. Does periodontitis reflect inflammation and malnutrition status in hemodialysis patients? Am J Kidney Dis 2006;47:815-22.

10. Gonçalves EM, Karam LALL, Milfont TS, Araújo MD, Santana JML, Lima DLF. Prevalência de periodontite em pacientes submetidos à hemodiálise. J Bras Nefrol 2007;29:115-9.

11. Slade GD, Offenbacher S, Beck JD, Heiss G, Pankow JS. Acute-phase inflammatory response to periodontal disease in the US population. J Dent Res 2000;79:4957.

12. Mattila KJ, Nieminen MS, Valtonen VV, Kesänieme YA, Syajälä SL, Jungell PS et al. Association between dental health and acute myocardial infarction. Br Med J 1989;298:779-82.

13. Pussinen PJ, Alfthan G, Tuomilehto J, Asikainen S, Jousilahti P. High serum antibody levels to Porphyromonas gingivalis predict myocardial infarction. Eur J Cardiovasc Prev Rehabil 2004;11:408-11.

14. Lima DLF, Moreira MMSM, Saba-Chujfi E, Pereira SLS, Soares Filho WA. Análise epidemiológica da doença periodontal em pacientes cardiopatas isquêmicos no Hospital de Messejana, na cidade de Fortaleza-Ceará. Periodontia 2004;14:17-21.

15. Scannapieco FA, Stewart EM, Mylotte JM. Colonization of dental plaque by respiratory pathogens in medical intensive care patients. Crit Care Med 1992;20:740-5.

16. Scannapieco FA, Mylotte JM. Relationships between periodontal disease and bacterial pneumonia. J Periodontol 1996;67:1114-22.

17. Offenbacher S, Katz V, Fertik G, Collins J, Boyd D, Maynor $G$ et al. Periodontal infection as a possible risk factor for preterm low birth weight. J Periodontol 1996;67(Suppl):1103-13.

18. Azevedo, ID. Periodontal Disease and low birth weight infants: a case control study [dissertation]. Natal (RN): Universidade Federal do Rio Grande do Norte; 2002.

19. Jeffcoat MK. Osteoporosis: a possible modifying factor in oral bone loss. Ann Periodontol 1998;3:312-21.

20. Yoshihara A, Seida Y, Hanada N, Miyazaki H. A longitudinal study of the relationship between periodontal disease and bone mineral density in community-dwelling older adults. J Clin Periodontol 2004;31:680-4.

21. Nishimura F, Takahashi K, Kurihara M, Takashiba S, Murayama Y. Periodontal disease as a complication of diabetes mellitus. Ann Periodontol 1998;3:20-9.

22. Taylor GW. Bidirectional interrelationships between diabetes and periodontal diseases: an epidemiologic perspective. Ann Periodontol 2001;6:99-112.

23. Guimarães MLR, Hilbert BJ, Hugo FN, Corso AC, Nocchi P, Padilha DMP. Impacto da perda dentária na qualidade de vida de idosos independentes. Scientia Medica 2005;15:30-3.

24. Bayraktar G, Kurtulus I, Duraduryan A, Cintan S, Kazancioglu R, Yildiz A et al. Dental and periodontal findings in hemodialysis patients. Oral Dis 2007;13:393-7.

25. Santos SC, Casati MZ, Sallum EA, Sallum AW. Conduta periodontal em pacientes com diabetes mellitus. Periodontia 2006;16:79-84.

26. Shultis WA, Weil EJ, Looker HC, Curtis JM, Shlossman M, Genco RJ et al. Effect of periodontitis on overt nephropathy and end-stage renal disease in type 2 diabetes. Diabetes Care 2007;30:306-11. 